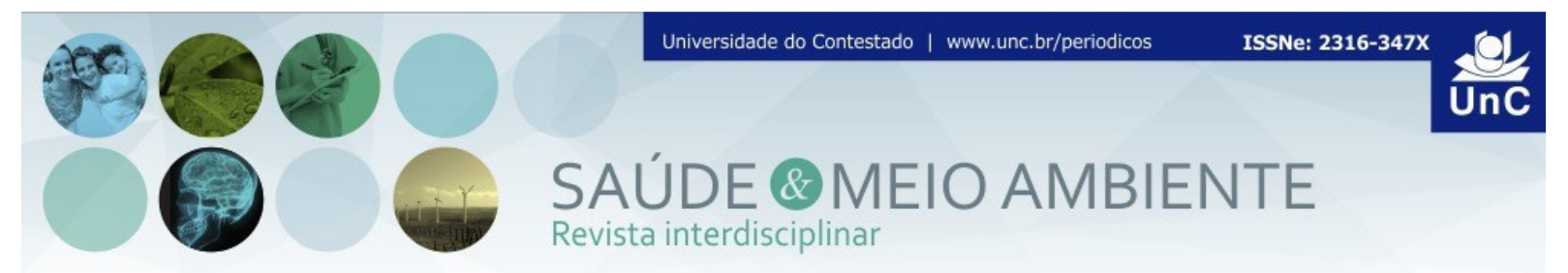

\title{
ECO-EPIDEMIOLOGIA DA LEISHMANIOSE TEGUMENTAR AMERICANA EM ITABUNA - BAHIA - BRASIL, 2001 A 2014
}

\section{ECO-EPIDEMIOLOGY OF AMERICAN TEGUMENTARY LEISHMANIASIS IN ITABUNA - BAHIA - BRAZIL, 2001-2014}

Adinailton Delmiro dos Santos ${ }^{1}$

Daniela Viana da Silva ${ }^{2}$

Samuel Martins de Jesus Branco ${ }^{3}$

Antônio José Costa Cardoso ${ }^{4}$

Sandra Adriana Neves Nunes ${ }^{5}$

\section{RESUMO}

Realizou-se estudo do tipo ecológico com o objetivo de investigar o perfil sociodemográfico e epidemiológico, a frequência de casos, os indicadores epidemiológicos e os aspectos eco-epidemiológicos da leishmaniose tegumentar americana em Itabuna. A metodologia usada foi o levantamento de dados secundários (DATASUS - 2001 a 2014). No período de 2001 a 2014 foram notificados 360 casos de leishmaniose tegumentar americana em Itabuna. Os dados foram analisados por meio de estatística descritiva e inferencial. A incidência média de leishmaniose tegumentar americana no município, de 2001-2014, foi de 12,1 casos/100.000 habitantes. Os resultados indicam que os indivíduos mais acometidos foram homens em idade economicamente ativa, com baixa escolaridade e residentes em área urbana. Os casos em sua maioria eram novos e a forma clínica mais prevalente foi a cutânea, sendo o critério de confirmação clínico-epidemiológico o mais frequente. $O$ teste $X^{2}$ evidenciou a existência de diferença significativa entre a frequência populacional e a frequência de casos da doença. Os indicadores

\footnotetext{
${ }^{1}$ Graduado em Bacharelado Interdisciplinar em Saúde. Atualmente cursa Medicina na Universidade Federal do Sul da Bahia. Bahia. Brasil. E-mail: adinailtonds@hotmail.com

2Possui graduação em Bacharelado em Ciências Biológicas e Bacharelado Interdisciplinar em Saúde, Mestrado em Genética e Biologia Molecular e Doutorado em Genética e Biologia Molecular.

Atualmente cursa Medicina na Universidade Federal do Sul da Bahia. Bahia. Brasil. E-mail:

danidvs@hotmail.com

${ }^{3}$ Possui graduação em Ciência Biológicas Licenciatura pela Universidade Estadual de Santa Cruz), Mestrado em Genética e Biologia Molecular pela Universidade Estadual de Santa Cruz e Doutorado em Genética e Biologia Molecular pela Universidade Estadual de Santa Cruz. Atualmente cursa Medicina na Universidade Federal do Sul da Bahia. Bahia. Brasil. E-mail: samuelbranco2005@hotmail.com

${ }^{4}$ Graduado em Medicina (FM/UFBA). Mestre em Saúde Comunitária (FM/UFBA) e Doutor em Saúde Pública com concentração na área de Planejamento e Gestão em Saúde (ISC/UFBA). Universidade Federal do Sul da Bahia, Instituto de Humanidades, Artes e Ciências do Campus Jorge Amado. Bahia. Brasil. ORCID: https://orcid.org/0000-0002-6085-3502. E-mail: antoniojccardoso@gmail.com

${ }^{5}$ Graduada em Psicologia pela Universidade Federal de Santa Catarina (UFSC). Mestre em Saúde pela Universidade de Greenwich, Inglaterra e Doutora em Psicologia pela UFSC. Professora adjunta da Universidade Federal do Sul da Bahia (UFSB) do Bacharelado Interdisciplinar em Saúde e do Programa de Pós-graduação em Estado e Sociedade. Bahia. Brasil. E-mail: psandranunes7@hotmail.com
} 
epidemiológicos mostraram a densidade espacial, a detecção da doença e a proporção de casos no município. No que se refere aos aspectos ecoepidemiológicos, foi possível estabelecer que a recente ocupação de áreas periurbanas no município, aliada a fatores ambientais, como clima, vegetação, acúmulo de matéria orgânica, parecem aumentar a vulnerabilidade da população a epidemias.

Palavras-chave: Perfil epidemiológico. Populações vulneráveis. Determinantes epidemiológicos. Leishmaniose cutânea.

\begin{abstract}
An ecological study was carried out to investigate the sociodemographic and epidemiological profile, the frequency of cases, the epidemiological indicators and the eco-epidemiological aspects of American cutaneous leishmaniasis in Itabuna. The methodology used was the collection of secondary data (DATASUS - 2001 to 2014). From 2001 to 2014, 360 cases of American cutaneous leishmaniasis were reported in Itabuna. The data were analyzed using descriptive and inferential statistics. The average incidence of American cutaneous leishmaniasis in the municipality, from 2001-2014, was 12.1 cases/100.000 inhabitants. The results indicate that the most affected individuals were men of economically active age, with low education and residents in urban areas. Most cases were new and the most prevalent clinical form was cutaneous, being clinical and epidemiological confirmation criterion the most frequent. The $X^{2}$ test showed the existence of a significant difference between the population frequency and the frequency of cases of the disease. Epidemiological indicators showed spatial density, disease detection and the proportion of cases in the municipality. With regard to eco-epidemiological aspects, it was possible to establish that the recent occupation of peri-urban areas in the municipality, combined with environmental factors, such as climate, vegetation, accumulation of organic matter, seem to increase the population's vulnerability to epidemics.
\end{abstract}

Keywords: Epidemiological profile. Vulnerable populations. Epidemiological determinants. Cutaneous leishmaniasis.

\title{
1 INTRODUÇÃO
}

A leishmaniose tegumentar americana é uma doença infectoparasitária causada por protozoários do gênero Leishmania que podem ser encontrados em hospedeiros vertebrados e, também, em vetores, os flebotomíneos, sendo esses os responsáveis por sua transmissão ${ }^{1}$. Essa doença é considerada um problema de saúde pública e está presente em todos os estados brasileiros ${ }^{2}$. 
Tem sido observada uma expansão geográfica da distribuição da doença em distintas áreas, tais como, garimpos, fronteiras agrícolas e periferia das cidades². Sua importância epidemiológica não decorre, entretanto, apenas desta ampliação territorial, mas das lesões que produz que, em estágios clínicos avançados, incapacitam e desfiguram o indivíduo, produzindo graves sequelas na esfera psicossocial ${ }^{1}$.

Quanto à produção científica, é válido mencionar Barata ${ }^{3}$ e Kawa et al. ${ }^{4}$, haja vista que dissertam sobre a importância dos determinantes sociais no entendimento do processo saúde-doença, sendo que o primeiro enfatiza o papel da epidemiologia social e o segundo a influência da organização do espaço na transmissão da doença. Quanto às publicações na microrregião Itabuna-llhéus, foram encontradas, por exemplo, uma dissertação $0^{5}$, uma tese ${ }^{6}$ e um artigo $^{7}$, todos focados na cidade de Ilhéus. Não há publicações referentes à situação epidemiológica da leishmaniose tegumentar americana no município de Itabuna, o que justifica a relevância do presente estudo em buscar preencher essa lacuna. Dessa forma, os objetivos desse estudo foram evidenciar o perfil sociodemográfico e epidemiológico da leishmaniose tegumentar americana em Itabuna; e descrever os aspectos eco-epidemiológicos da doença no município.

No intuito de analisar os aspectos eco-epidemiológicos da leishmaniose tegumentar americana no município de Itabuna, buscou-se refletir sobre os determinantes sociais ao responder as indagações propostas por Barata ${ }^{3}$ (p. 11): "O que coloca a população em risco de epidemia? Que características populacionais aumentam a vulnerabilidade a epidemias?".

\section{MATERIAL E MÉTODOS}

O entendimento da eco-epidemiologia perpassa pela abordagem das "caixas chinesas"8, na qual há a possibilidade de análise em distintos níveis hierárquicos que interagem e se encaixam. Desta forma, é possível investigar os determinantes envolvidos no processo saúde-doença em uma escala ampliada de complexidade, abarcando não apenas variáveis fisiológicas, mas, também, ambientais.

É válido mencionar que a eco-epidemiologia difere da abordagem multicausal por considerar os processos em um âmbito populacional, sendo necessário, por isso, incluir os determinantes sociais ${ }^{3}$. Os determinantes sociais são os elementos que conectam as condições de vida e trabalho à situação de saúde interferindo no bem-estar dos indivíduos. Tais fatores podem englobar variáveis econômicas, políticas, culturais, étnicas, comportamentais, sociais e psicológicas ${ }^{9}$.

Foram realizadas buscas nos bancos de dados da Scielo ${ }^{10}$ e Google Acadêmico ${ }^{11}$, acerca dos determinantes sociais e das mudanças ocorridas, ao longo do tempo, que possam estar relacionadas com a eco-epidemiologia da leishmaniose no município. 


\section{1 ÁREA DE ESTUDO}

Itabuna está situada em $14^{\circ} 48^{\prime}$ Latitude Sul e $39^{\circ} 18^{\prime}$ Longitude Oeste, a 433 $\mathrm{km}$ de Salvador na mesorregião geográfica Sul baiana. O município integra a Região Cacaueira da microrregião Ilhéus-Itabuna, no território de identidade Litoral Sul, inserida no bioma da Mata Atlântica. Apresenta clima tropical quente e úmido, sem estação seca, com umidade relativa do ar superior a $80 \%$ e média de pluviosidade de $1300,3 \mathrm{~mm}^{12}$. Possuía em 2016, segundo dados do $\mathrm{IBGE}^{13}$, uma população de 220.386 habitantes em uma unidade territorial de $401,028 \mathrm{~km}^{2}$.

\subsection{COLETA DE DADOS}

Foi realizado um estudo ecológico do tipo série temporal, quantitativo e retrospectivo. Os dados epidemiológicos sobre a leishmaniose tegumentar americana, de 2001 a 2014 em Itabuna, foram coletadas no banco de dados do Datasus $^{14}$. As variáveis analisadas foram: sexo, idade, raça, escolaridade, zona de residência, tipo de entrada, forma clínica, critério de confirmação, evolução do caso e mês de notificação. Além disso, também foram coletados dados referentes ao número de habitantes por sexo no município de Itabuna.

\subsection{ANÁLISE ESTATÍSTICA}

Para a análise de dados foram empregadas a estatística descritiva e inferencial. Para fins de análise descritiva foram calculados a amplitude, a média e o desvio padrão médio, utilizando os dados de mês de notificação, do período de 2007-2014, visto que não há o registro dos anos anteriores. Já no que diz respeito à análise inferencial, o software Bioestat ${ }^{15}$, versão 5.3 , foi utilizado para a realização de testes não paramétricos de $X^{2}$, para avaliar as diferenças na frequência de casos de leishmaniose tegumentar americana que foram notificados por sexo de 20012014 , considerando $p<0,05$. Os dados populacionais e a incidência da doença por sexo foram analisados por porcentagem, permitindo assim, compará-los.

\subsection{CÁLCULO DOS INDICADORES EPIDEMIOLÓGICOS}

Os indicadores epidemiológicos calculados foram: densidade de casos (número de casos referente à forma clínica, dividido pela área do município e 
multiplicado por 100); proporção de casos conforme a forma clínica (número de casos referente a forma clínica, dividido pelo número total de casos e multiplicado por 100); e coeficiente geral de detecção (número de casos, dividido pela população e multiplicado por 100.000 habitantes $)^{16}$. Também foi calculada a incidência média de leishmaniose tegumentar americana no período de 2001-2014 (média de casos notificados, multiplicado por 100.000 habitantes e dividido pela média da população).

\section{RESULTADOS}

\subsection{PERFIL EPIDEMIOLÓGICO}

No período de 2001 a 2014 foram notificados 360 casos de leishmaniose tegumentar americana em Itabuna. Desses, $62,5 \%$ correspondiam ao sexo masculino e $37,5 \%$ ao sexo feminino. Os indivíduos mais acometidos eram pardos $(54,4 \%)$, na faixa etária de 20 a 59 anos $(56,7 \%)$, com baixa escolaridade $(63,9 \%)$, tendo cursado no máximo o ensino fundamental, e residentes na área urbana $(76,1 \%)$.

No que concerne à infecção por leishmaniose tegumentar americana, 88,9\% dos casos foram novos, 3,6\% foram recidivas e 7,5\% referem-se a informações perdidas. A forma clínica mais prevalente foi a cutânea, abarcando $88,9 \%$ dos casos.

Os dados pertencentes aos critérios de confirmação indicaram que 56,9\% dos casos foram confirmados por via clínica-epidemiológica, $33,1 \%$ via clínicalaboratorial, e 10\% referem-se a informações perdidas. Os casos evoluíram em cura $(45,8 \%)$, abandono $(14,7 \%)$, transferência $(1,2 \%)$ e óbito $(0,8 \%)$, ressaltando ainda, que $37,5 \%$ dos dados desta variável referem-se a informações perdidas.

\subsection{ANÁLISE ESTATÍSTICA}

No período de 2007 a 2014, a amplitude de casos foi de 7 a 20 notificações. O mês que apresentou a maior incidência foi setembro, com 20 notificações, acompanhado de abril, com 18 notificações. Os meses com as menores incidências foram dezembro e maio, com sete e oito notificações, respectivamente (Figura 1). A média de notificações por mês foi de 11,75 , enquanto o desvio padrão médio foi de 3,21 . 
Figura 1 - Mês de notificação de leishmaniose tegumentar americana, no período de 2007-2014, em Itabuna - Bahia.

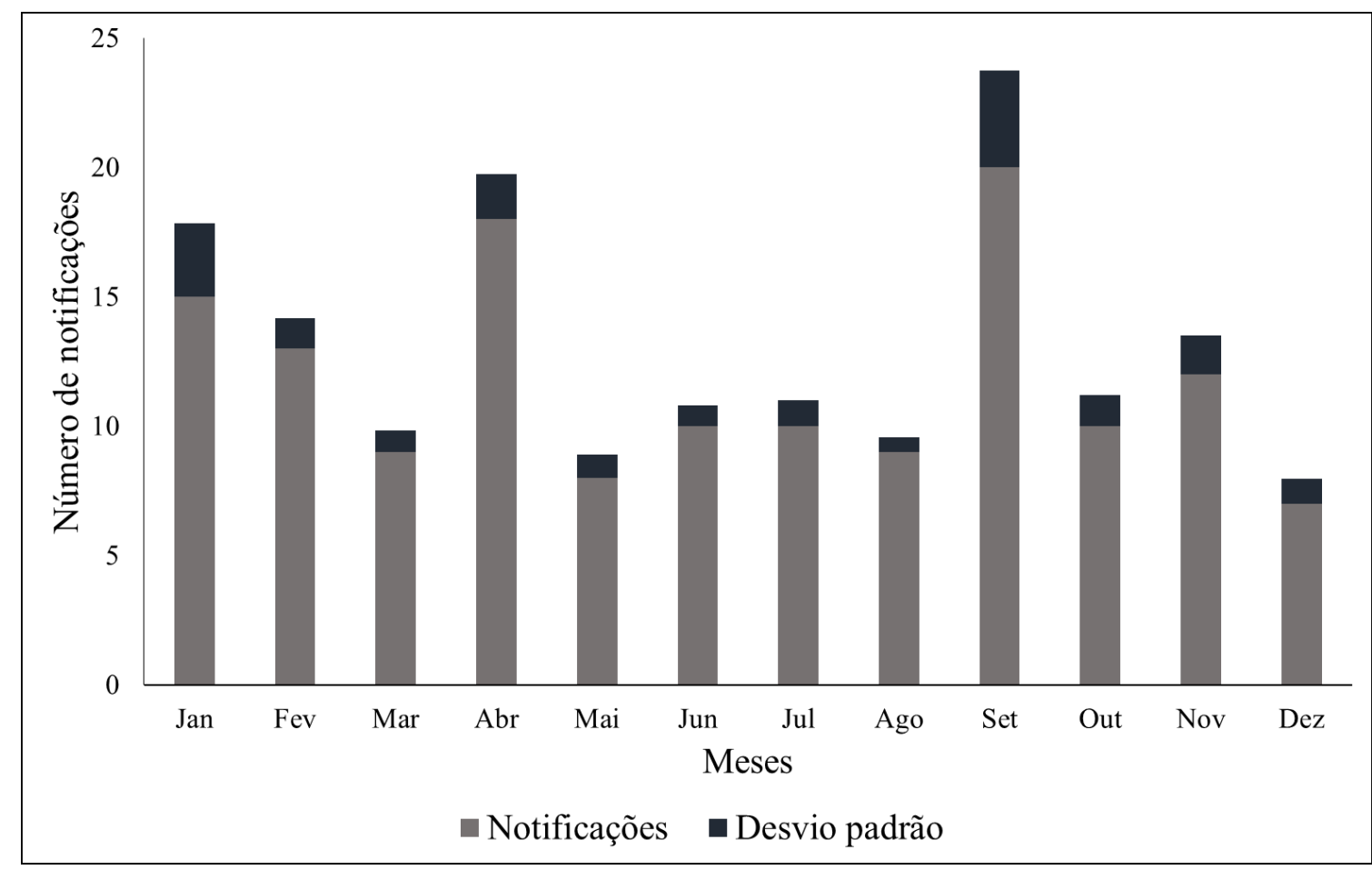

Fonte: Ministério da Saúde/SVS - Sistema de Informação de Agravos de Notificação - Sinan.

A população média de Itabuna - Bahia, de 2001 a 2014, era composta por $51,8 \%$ de mulheres e $48,2 \%$ de homens. O teste não paramétrico de $x^{2}$ evidenciou a existência de diferenças entre frequência de casos de leishmaniose tegumentar americana que foram notificados por sexo de 2001-2014 e a frequência populacional, considerando $p<0,05$ (Figura 2). 
Figura 2 - Frequência de leishmaniose tegumentar americana por sexo, em Itabuna - Bahia, de 2001 a 2014. A: diferença na frequência de casos de leishmaniose no sexo masculino. B: diferença na frequência de casos de leishmaniose tegumentar americana no sexo feminino.

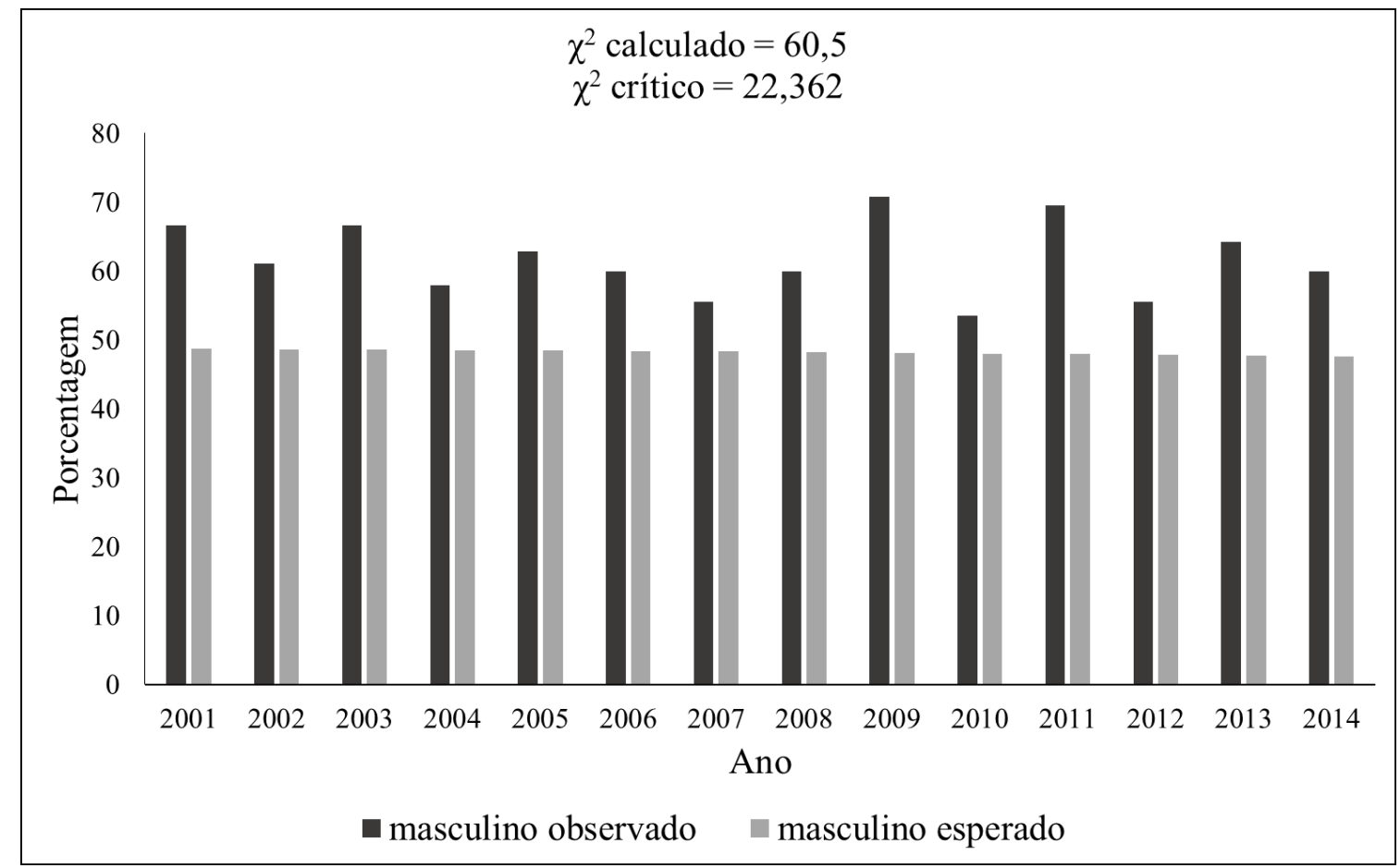

A

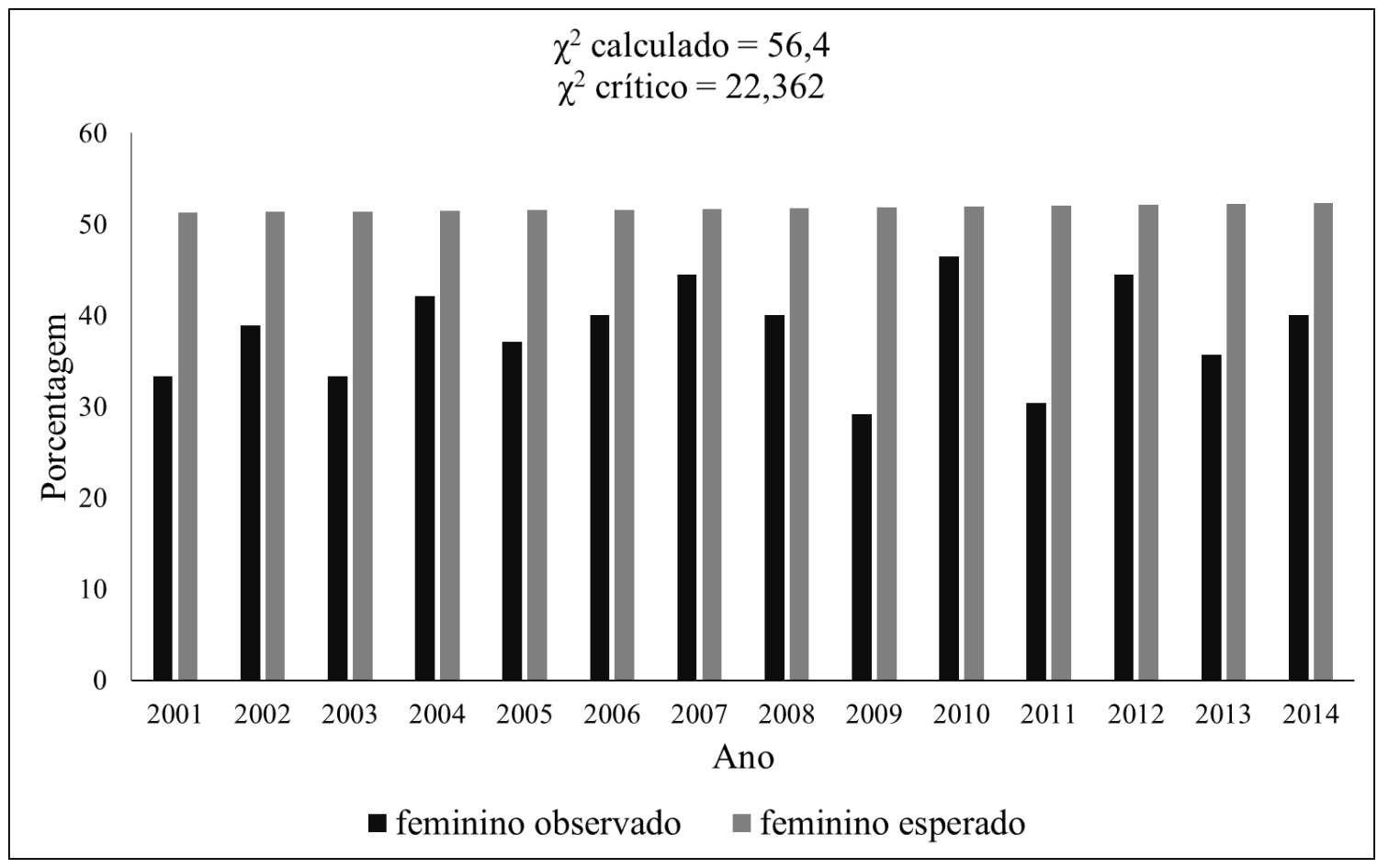

B

Fonte: Ministério da Saúde/SVS - Sistema de Informação de Agravos de Notificação - Sinan. 


\subsection{INDICADORES EPIDEMIOLÓGICOS}

Os indicadores epidemiológicos da densidade espacial, proporção de casos e detecção da doença no município de Itabuna estão apresentados na Tabela 1 . A incidência média de leishmaniose tegumentar americana no município, de 2001 2014 , foi de 12,1 casos detectados/100.000 habitantes.

Tabela 1 - Indicadores epidemiológicos de leishmaniose tegumentar americana, em Itabuna - Bahia, de 2001-2014.

\begin{tabular}{cccccc}
\hline $\begin{array}{c}\text { Ano do } \\
\text { Diagnóstico }\end{array}$ & \multicolumn{2}{c}{$\begin{array}{c}\text { Densidade de casos } \\
\left(\mathrm{km}^{2}\right)\end{array}$} & \multicolumn{2}{c}{ Proporção de casos } & $\begin{array}{c}\text { Coeficiente geral de } \\
\text { detecção }(100.000 \\
\text { habitantes })\end{array}$ \\
\cline { 2 - 5 } & Cutânea & Mucosa & Cutânea & Mucosa & \\
\hline 2001 & 7,98 & 0,25 & 71,11 & 2,22 & 21,97 \\
2002 & 7,73 & 0,50 & 86,11 & 5,56 & 17,46 \\
2003 & 10,22 & 0,75 & 91,11 & 6,67 & 21,68 \\
2004 & 8,98 & 0,50 & 94,74 & 5,26 & 18,19 \\
2005 & 7,73 & 1,00 & 88,57 & 11,43 & 16,66 \\
2006 & 4,74 & 0,25 & 95,00 & 5,00 & 9,46 \\
2007 & 2,24 & 0,00 & 100,00 & 0,00 & 4,24 \\
2008 & 1,99 & 0,50 & 80,00 & 20,00 & 4,68 \\
2009 & 5,74 & 0,25 & 95,83 & 4,17 & 11,18 \\
2010 & 6,48 & 0,50 & 92,86 & 7,14 & 12,98 \\
2011 & 5,49 & 0,25 & 95,65 & 4,35 & 10,62 \\
2012 & 3,49 & 1,00 & 77,78 & 22,22 & 8,28 \\
2013 & 3,24 & 0,25 & 92,86 & 7,14 & 6,42 \\
2014 & 3,74 & 0,00 & 100,00 & 0,00 & 6,85 \\
\hline
\end{tabular}

Fonte: Ministério da Saúde/SVS - Sistema de Informação de Agravos de Notificação - Sinan.

Elaboração própria.

\subsection{ASPECTOS ECO-EPIDEMIOLÓGICOS}

Visando responder "o que coloca a população em risco de epidemia e quais as características populacionais que aumentam a vulnerabilidade da população"3, adentramos nos aspectos ecológicos e sociais que contribuem para o desenvolvimento e manutenção do ciclo da doença. Os referenciais teóricos foram utilizados para construir os determinantes de Itabuna ${ }^{12 ; 21 ; 22 ; 23 ; 24 .}$

Sugere-se, portanto, que variáveis como clima, vegetação, acúmulo de matéria orgânica e recente ocupação de áreas periurbanas, integram os determinantes ecológicos e sociais envolvidos nos processos de risco e/ou 
vulnerabilidade a epidemia e transmissão da leishmaniose tegumentar americana em Itabuna - Bahia.

\section{DISCUSSÃO}

O perfil epidemiológico dos indivíduos acometidos por leishmaniose tegumentar americana em Itabuna (em sua maioria homens em idade economicamente ativa, com baixa escolaridade e residentes em área urbana) corrobora com o perfil encontrado para a mesma doença em outras localidades. Guerra et al. ${ }^{2}$ identificaram a prevalência de casos entre homens com idade de 20 a 39 anos em zona rural de Manaus; Lima et al. ${ }^{17}$ indicaram o predomínio de indivíduos do sexo masculino, com idade entre 21 e 40 anos e residentes em área urbana no noroeste do Paraná; Naiff Júnior et al. ${ }^{18}$ mostraram que o maior número de casos estava presente no sexo masculino, com idade entre 20 e 39 anos, em Rio Preto da Eva no Amazonas; e Nobres et al. ${ }^{19}$ verificaram que a quantidade de casos era preponderante em homens com idade entre 20 e 49 anos no norte de Mato Grosso.

O perfil epidemiológico sugere que os indivíduos acometidos estão em idade economicamente ativa, e por não possuírem alta escolaridade, ocupam atividades laborais em áreas onde a veiculação da doença está presente, tais como, em atividades agrícolas. Nesse contexto, Lima et al. ${ }^{17}$ inferem que a maior incidência da doença está relacionada ao trabalho rural em áreas próximas a matas, e Naiff Júnior et al. ${ }^{18}$ denotam que o aumento na incidência está associado ao número de indivíduos em assentamento agrários, os quais estão localizados nas adjacências, em contato direto, com as florestas.

Em relação à maior prevalência da forma clínica cutânea da leishmaniose tegumentar americana em Itabuna, este achado está em consonância com os apresentados por outros municípios ${ }^{18,19}$. Além disso, o baixo índice de recidivas e a porcentagem dos casos que evoluíram para cura $(45,8 \%)$ sugerem que o tratamento da doença está sendo efetivo; no entanto, devido à quantidade de informações perdidas no que concerne à evolução dos casos $(37,5 \%)$, não é possível inferir sobre tal fato com precisão.

A leishmaniose tegumentar americana é uma doença de notificação compulsória. O quadro clínico das lesões evolui após um período de incubação que oscila de dez dias a três meses ${ }^{1}$. Desta forma, mesmo tendo os dados referentes aos meses de notificação dos casos, não é possível predizer, com certeza, em quais meses os indivíduos tiveram contato com os vetores da doença. Ainda assim, conhecendo o cenário agrícola do município, o qual está relacionado com a cultura do cacau, sugere-se que o contato com os agentes transmissores possa ocorrer durante o manejo e colheita da cultura.

A população de Itabuna, nesse período de 2001 a 2014, possuía mais mulheres do que homens; no entanto, foi observado maior frequência de casos no 
sexo masculino. O teste não paramétrico evidenciou uma diferença significativa entre a frequência populacional e a frequência de casos. Esta diferença pode ser explicada devido à divisão sexual do trabalho no campo, a qual historicamente distribui às ocupações por gênero, cabendo aos homens as etapas braçais. Santos e Garcia $^{20}$ enfatizam a discussão sobre a divisão de trabalho segundo gênero, relatando sobre a luta das mulheres pela igualdade e o processo de construção e ocupação de espaços no campo.

No que concerne aos indicadores epidemiológicos, foi possível discernir sobre a extensão da doença em Itabuna, provendo informações que podem embasar o planejamento de ações, no âmbito municipal, para implementar medidas que visem o controle do agravo. Ressalta-se que o coeficiente de detecção, o qual expressa o risco de adquirir a doença ${ }^{16}$, oscilou, não representando um padrão ou um ciclo, mas houve uma queda ao longo do tempo. Sugere-se, portanto, que outros fatores, não apenas biológicos, mas ambientais e sociais também estejam associados com a transmissão da doença.

Nesse contexto, é de fundamental importância conhecer os aspectos sociais e ecológicos de Itabuna, e para isso, é necessário explorar a história do município. Silva ${ }^{21}$ representa Itabuna em suas origens como local de mata inóspita repleta de animais ferozes, embora já houvesse populações indígenas e colonos. Com o passar do tempo, a identidade local se solidificou na figura do trabalhador rural, o qual derrubou florestas, acumulou riquezas e construiu fazendas de cacau. A cacauicultura foi o símbolo de riqueza e prosperidade. Os coronéis do cacau mantinham o poder perante as decisões locais, sendo, por isso, figuras de prestígio. Cidades, vilas, estradas e escolas foram construídas com o dinheiro oriundo do cacau.

Além disso, essa cultura também beneficiava o meio ambiente, visto que a Mata Atlântica era preservada pelo sistema tradicional de cultivo, a cabruca, a qual mantinha o estrato arbóreo. No entanto, em 1989 houve a dispersão de uma doença fúngica, a vassoura-de-bruxa, instalando uma enorme crise econômica. Com isso, a realidade local se modificou, os produtores se endividaram, as plantações foram abandonadas e o desemprego rural e urbano foi ampliado ${ }^{22}$.

A população de Itabuna sentiu os impactos da pobreza e da miséria, aumentando a quantidade de moradores nas periferias da cidade. Contudo, no intuito de remediar os reflexos da crise, o município diversificou sua base econômica por meio da instalação de indústrias e intensificou o comércio ${ }^{23}$; a maior parcela da sua população passou a viver na zona urbana, trazendo à tona, no cenário urbano, os problemas sociais oriundos das ocupações desordenadas, tais como, acúmulo de resíduos orgânicos e de entulhos, desabamento de residências e, em períodos de chuvas, a ocorrência de inundações ${ }^{24}$.

Quanto aos aspectos ecológicos, o clima tropical quente e úmido, temperatura média anual em torno de $23,6^{\circ} \mathrm{C}$, umidade relativa do ar superior a $80 \%$ e bioma da Mata Atlântica ${ }^{12}$ são favoráveis a proliferação dos mosquitos vetores da doença. Além disso, os aspectos sociais, tais como a recente ocupação de áreas periurbanas (área urbana próximo de áreas rurais) e acúmulo de matéria orgânica, em 
consonância com os fatores ambientais e de tratos culturais (o sistema de cultivo de cabruca favoreceu a conservação de regiões de mata), são fatores facilitadores do contato dos indivíduos com os vetores transmissores da doença. Ximenes et al. ${ }^{25}$ corroboram a relação entre fatores ambientais, a proliferação de vetores e a saúde dos seres humanos em estudo realizado no Rio Grande do Norte.

Desta forma, as respostas para as duas indagações propostas ("O que coloca a população em risco de epidemia? Que características populacionais aumentam a vulnerabilidade a epidemias?" 3 ) estão embasadas em aspectos ecológicos e sociais que norteiam o cotidiano dos indivíduos. A população está em risco sempre que mantem contato com os agentes transmissores da doença. $\mathrm{E}$ as características populacionais que aumentam a vulnerabilidade a epidemias são: aumento populacional em áreas periurbanas; atividades de caça, as quais aumentam o contato com animais reservatórios; atividade laboral rural; e baixa escolaridade e consequente ausência de informação acerca de profilaxia e tratamento efetivo para a doença.

\section{CONCLUSÃO}

Partindo dos pressupostos apresentados nesse estudo, foi possível sugerir os determinantes sociais que estão associados com a leishmaniose tegumentar americana no município, assim como, estabelecer o perfil sociodemográfico da população acometida. Essas informações são de extrema importância para a compreensão da realidade epidemiológica de Itabuna e podem ser utilizadas para a elaboração de planos de ação que visem o controle e manejo da doença.

Fontes de financiamento: Este estudo não recebeu financiamento.

Conflito de interesse: Os autores declaram que não há conflito de interesse.

\section{REFERÊNCIAS}

1. Gontijo B, Carvalho MLR. Leishmaniose tegumentar americana. Rev Soc Bras Med Trop 2003; 36:71-80.

2. Guerra JAO, Ribeiro JAS, Coelho LIARC, Barbosa MGV, Paes MG. Epidemiologia da leishmaniose tegumentar na Comunidade São João, Manaus, Amazonas, Brasil. Cad. Saúde Pública 2006; 22:2319-2327.

3. Barata RB. Epidemiologia social. Rev Bras Epidemiol 2005; 8:7-17. 
4. Kawa H, Sabroza PC, Oliveira RM, Barcellos C. A produção do lugar de transmissão da leishmaniose tegumentar: o caso da Localidade Pau da Fome na cidade do Rio de Janeiro, Brasil. Cad. Saúde Pública 2010; 26:1495-1507.

5. Souza YB. Leishmaniose tegumentar americana no município de llhéus-BA: caracterização de casos humanos e fatores de risco associados [dissertação]. Viçosa: Universidade Federal de Viçosa; 2007.

6. Carvalho SMS. Caracterização da transmissão de Leishmaniose Tegumentar Americana no município de Ilhéus, Zona da Mata do Estado da Bahia [tese]. Recife: Centro de Pesquisas Aggeu Magalhães; 2010.

7. Carvalho SMS, Santos PRB, Lanza H, Brandão-Filho SP. Diversidade de flebotomíneos no Município de Ilhéus, Bahia. Epidemiol Serv Saúde 2010; 19:239-244.

8. Susser M, Susser E. Choosing a Future for Epidemiology: II. From Black Box to Chinese Boxes and Eco-Epidemiology. Amer Jour Publ Health 1996; 86:674-77.

9. Buss PM, Pellegrini Filho A. A saúde e seus determinantes sociais. PHYSIS: Rev Saúde Coletiva 2007; 17:77-93.

10. Scielo [Internet]. Library collection. Disponível em: www.scielo.br.

11. Google Acadêmico [Internet]. Disponível em: scholar.google.com.br.

12. PMI/UESC (Prefeitura Municipal de Itabuna / Universidade Estadual de Santa Cruz). Anuário Estatístico de Itabuna: ano base 2011. Itabuna: PMI/UESC; 2013.

13. IBGE (Instituto Brasileiro de Geografia e Estatística) [Internet]. Cidades 2016. Disponível em: http://www.cidades.ibge.gov.br/xtras/perfil.php?lang=\&codmun=2 91480.

14. Datasus [Internet]. Informações de Sáude, Epidemiológicas e Morbidade. Disponível em: http://datasus.saude.gov.br/ informacoes-desaude/tabnet/epidemiologicas-e-morbidade.

15. Ayres M, Ayres Jr M, Ayres DL, Santos AAS. Bioestat: aplicações estatísticas nas áreas das Ciências Biomédicas. Versão 5.3. Bélem: Sociedade Civil Mamirauá; 2007.

16. Andrade TAS, Soares FCS, Ramos JVA, Faustino MAG. Perfil epidemiológico dos casos notificados de leishmaniose tegumentar americana no município de Igarassu (PE) no período de 2008 a 2010. Scire Salutis 2012; 2:5-15.

17. Lima MVN, Oliveira RZ, Lima AP, Felix MLO, Silveira TGV, Rossi RM, Teodoro U. Atendimento de pacientes com leishmaniose tegumentar americana: avaliação nos serviços de saúde de municípios do noroeste do Estado do Paraná, Brasil. Cad. Saúde Pública 2007; 23:2938-2948. 
18. Naiff Júnior RD, Pinheiro FG, Naiff MF, Souza IS, Castro LM, Menezes MP, Franco AMR. Estudo de uma série de casos de leishmaniose tegumentar americana no município de Rio Preto da Eva, Amazonas, Brasil. Rev Patol Trop 2009; 38:103-114.

19. Nobres ES, Souza LA, Rodrigues DJ. Incidência de leishmaniose tegumentar americana no norte de Mato Grosso entre 2001 e 2008. Acta Amazonica 2013; 43:297-304.

20. Santos FF, Garcia MF. A luta da mulher pela igualdade no campo: contradições e tensões no sistema capitalista. Rev Interface 2015; 296-303.

21. Silva AO. As origens de Itabuna: o mito e a história. Rev Mosaico 2010; 2:96119.

22. Rocha LB. A região cacaueira da Bahia - dos coronéis à vassoura-de-bruxa: saga, percepção, representação. Ilhéus: Editus; 2008.

23. Rocha LB. O centro da cidade de Itabuna: trajetória, signos e significados. Ilhéus: Editus; 2003.

24. Hora SB, Gomes RL. Mapeamento e avaliação do risco a inundação do Rio Cachoeira em trecho da área urbana do Município de Itabuna/BA. Soc \& Nat 2009; 21:57-75.

25. Ximenes MFFM, Silva VPM, Queiroz PVS, Rego MM, Cortez AM, Batista LMM, Medeiros A, Jeronimio SMB. Flebotomíneos (Diptera: Psychodidae) e Leishmanioses no Rio Grande do Norte, Nordeste do Brasil - Reflexos do Ambiente Antrópico. Neotrop Entomol 2007; 36:128-137.

Artigo recebido em: 02/06/2020

Artigo aprovado em: 29/09/2020

Artigo publicado em: 26/10/2020 